\title{
Mature Ganglioneuroma
}

National Cancer Institute

\section{Source}

National Cancer Institute. Mature Ganglioneuroma. NCI Thesaurus. Code C42065.

A gang lioneuroma characterized by the presence of mature gang lion cells and a mature Schwannian stroma. 